\title{
Multiple tendons of the additional belly of flexor pollicis longus in the carpal tunnel: Embryological perspective and their clinical significance
}

\author{
Ravindra Swamy Shantakumar, Srinivasa Rao Sirasanagandla*, \\ Satheesha Badagabettu Nayak, Mohandas Rao Kappettu Gadahad, \\ Shiroor Nagabhushan Somayaji, Naveen Kumar \\ Melaka Manipal Medical College (Manipal Campus), Manipal University, Manipal, India; \\ *Corresponding Author: seenaih.anat@gmail.com
}

Received 30 April 2013; revised 2 June 2013; accepted 10 June 2013

Copyright (C) 2013 Ravindra Swamy Shantakumar et al. This is an open access article distributed under the Creative Commons Attribution License, which permits unrestricted use, distribution, and reproduction in any medium, provided the original work is properly cited.

\begin{abstract}
Although the flexor pollicis longus is known to show the additional head of the origin, the occurrence of its additional tendons in the carpal tunnel are seldom reported. The presence of such additional tendons in the carpal tunnel cannot be overlooked during the radiological and surgical procedures in this region. Herein, we report a rare case of additional muscle belly of flexor pollicis longus. The additional muscle belly after a short course divided into three tendons. All three tendons entered the carpal tunnel along with flexor pollicis longus, passing deep to the flexor retinaculum. Within the carpal tunnel, two of these tendons fused and terminated by merging with the undersurface of the flexor retinaculum. The third tendon terminated by joining the flexor digitorum superficialis tendon for the index finger, in the palm. An additional slip of the first lumbrical muscle took origin from the third tendon of the additional muscle belly of flexor pollicis longus. Further, the embryological basis and clinical significance of current case is discussed.
\end{abstract}

Keywords: Flexor Pollicis Longus; Additional Muscle Belly; Flexor Retinaculum; Carpal Tunnel; First Lumbrical

\section{INTRODUCTION}

The flexor pollicis longus muscle (FPL) is one of the deep muscles of the forearm. It originates from the grooved anterior surface of the radius and from the adjacent interosseous membrane and gets inserted to the base of the distal phalanx of the thumb [1]. In the past, the occurrence of accessory head of the FPL has been reported frequently [2-7]. Accessory head has been noted arising from the lateral or more rarely from the medial border of the coronoid process or also from the medial epicondyle of the humerus [3]. Frequently, accessory head is also called as Gantzer's muscle, or occasional head [3]. Though FPL is known to present the accessory head frequently, the occurrence of its additional muscle bellies and tendons is very rare. The presence of anomalous tendinous slip of FPL [2], unusual origin of first lumbrical from this anomalous tendinous slip [8] and insertion of this slip to the flexor digitorum profundus muscle (FDP) [9] have been noted. In the present case, we report an additional muscle belly of FPL having unique mode of insertion. The embryological basis and clinical significance of variant additional belly of FPL are discussed.

\section{CASE REPORT}

During regular dissection classes for Medical undergraduates, we came across a rare case of additional belly of FPL, in an approximately 55-year-old male cadaver. The FPL originated from anterior surface of the radius and from the adjacent interosseous membrane. It ran on the radial side of the forearm as a normal sized tendon, entered the palm by passing deep to the flexor retinaculum and finally got inserted to the base of the distal phalanx of the thumb. An additional muscle belly was originating from the ulnar side of the FPL, near its upper end. The additional muscle belly coursed between the FPL and FDP muscles and then divided into three tendons 
(Figure 1). All three aberrant tendons entered the carpal tunnel along with FPL passing deep to the flexor retinaculum. Within the carpal tunnel, two of these tendons fused and terminated by merging with the undersurface of the flexor retinaculum (Figure 2). Remaining third aberrant tendon, unlike other tendons entered the palm and finally terminated by blending with the flexor digitorum superficialis (FDS) tendon for the index finger. The first lumbrical muscle presented bipennate arrangement as one of its slip arose from the radial side of FDP tendon of the index finger and another slip arose from the third tendon of the additional belly of FPL. The tendon of first lumbrical was inserted to the radial side of the base of the proximal phalanx and dorsal digital expansion (Figure 3). It received nerve supply from the median nerve.

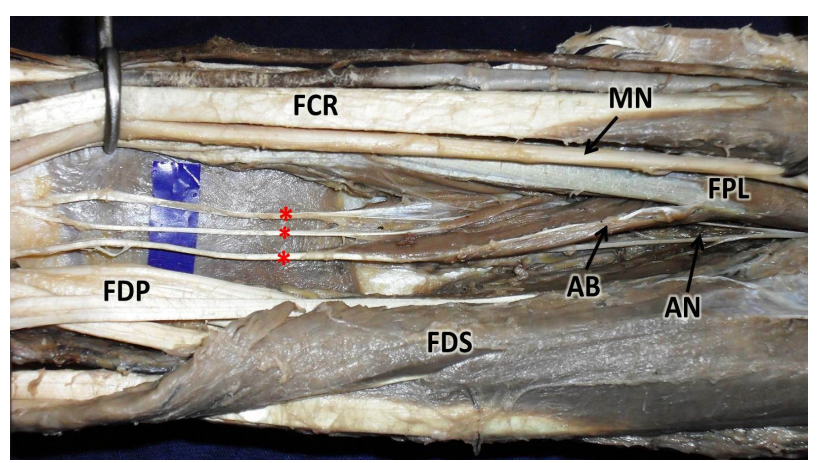

Figure 1. Dissection of flexor compartment of the right forearm showing the additional muscle belly (AB) of flexor pollicis longus muscle (FPL). Note the three tendons of additional (asterisks) belly running distally between the FPL and Flexor digitorum profundus muscle (FDP). (AN: anterior interoosseus nerve, FDS: flexor digitorum superficialis, FCR: flexor carpi radialis, $\mathrm{MN}$ : median nerve).

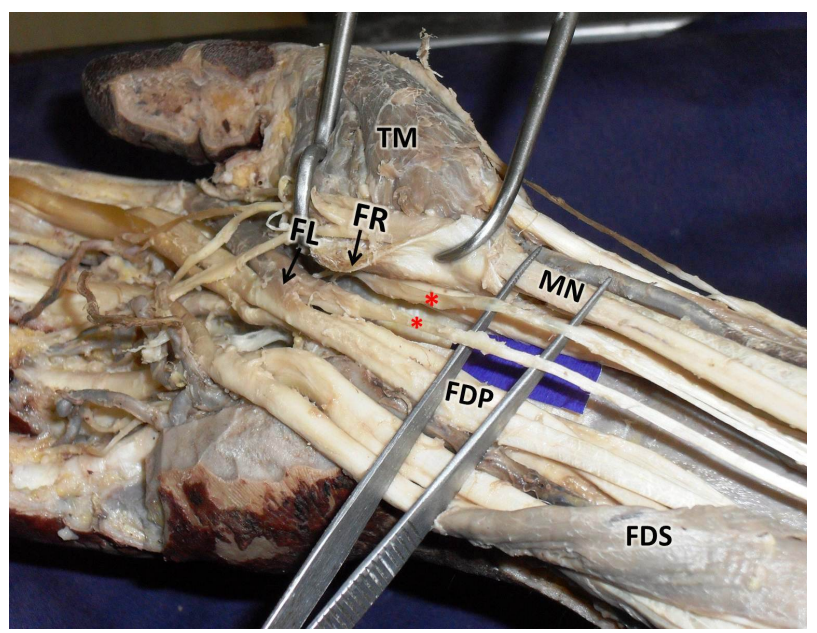

Figure 2. Dissection of flexor compartment of the right forearm and palm showing the additional tendons of (asterisks) flexor pollicis longus muscle (FPL), in the carpal tunnel. Insertion of one of the tendon to the undersurface of flexor retinaculum (FR) is also seen. (FDS: flexor digitorum superficialis,

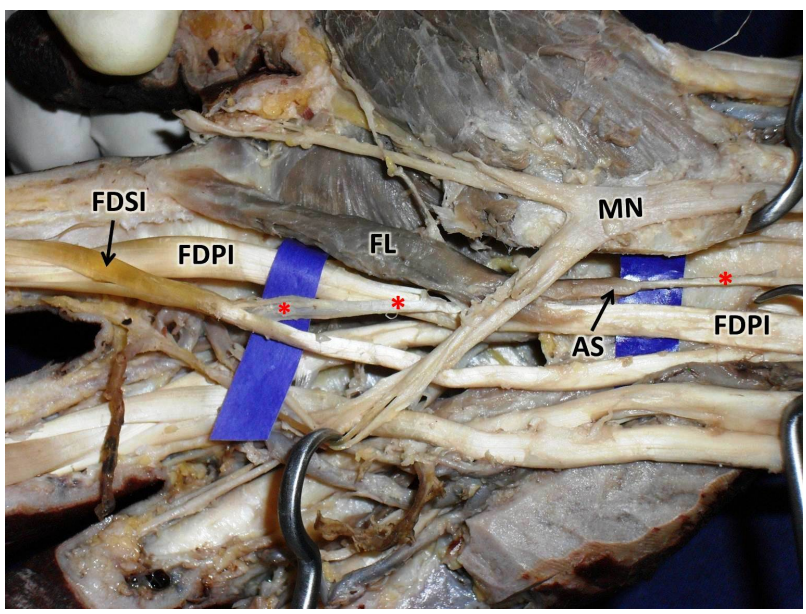

Figure 3. Dissection of flexor compartment of the forearm and palm showing the course of one of the additional tendons of (asters) flexor pollicis longus muscle, in the carpal tunnel. Note the insertion of this additional tendon (asters) to the tendon of flexor digitorum superficialis for index finger (FDSI). The additional slip (AS) of origin of first lumbrical (FL) from the additional tendon (asterisks) of FPL is also seen. (FDPI: Flexor digitorum profundus for index finger, MN: median nerve, FL: first lumbrical).

FDP: Flexor digitorum profundus muscle, FCR: flexor carpi radialis, MN: median nerve, TM: thenar muscles, FL: first lumbrical).

\section{DISCUSSION}

The occurrence of the additional head of FPL is not uncommon [6]. In most of the studies, the prevalence was found to be more than 50\% [2-7]. The reported cases in past also demonstrates that the accessory heads frequently occur bilaterally rather than unilaterally [7]. Though, FPL frequently shows the accessory heads, the occurrence of its additional muscle bellies and tendons is very rare. Macalister [8] and Wood [2] have noted the unusual origin of first lumbrical muscle from the additional tendinous slip of FPL. Kumar et al. have reported the cases of presence of an additional muscle belly from the FPL [10]. In their study, the tendon after receiving another accessory tendon from the FDP, finally inserted to the FDP tendon for the index finger. Linburg and Comstock have also observed such connection between the FPL and FDP in four of their patients [9]. They have noted such anatomical variation with the incidence of $31 \%$ cases unilaterally and $14 \%$ cases bilaterally. Presence of such anomaly may cause chronic tenosynovitis. The occurrence of this variant was also studied in formalin embalmed cadavers and noted in $25 \%$ of cases unilaterally and $6 \%$ of cases bilaterally [9]. Contrary to previous reports, we report a unique case of anomalous tendons of FPL having unusual mode of insertion to the tendon of FDS for the index finger in the palm and to 
undersurface of the flexor retinaculum in the carpal tunnel.

The embryological basis for the existence of additional muscles in the flexor compartment of the forearm may be attributed to the incomplete separation of the flexor mass [11]. During intrauterine life, the flexor muscles of the forearm develop from the flexor mass. This mass subsequently divides into superficial and deep layers. The deep layer of the flexor muscle mass forms the FPL, FDP and FDS [12]. The additional muscle belly observed in the present case might have occurred due to incomplete cleavage of the flexor mass.

Lumbricals frequently shows variations in their origin, insertion and number [13]. Lumbricals usually take origin from the tendons of the FDP. In the past, their unusual origin from flexor retinaculum, FDS or FDP has been reported. Occurrence of additional slip of origin of first lumbrical has been observed and noted to occur from FPL, FDS, first metacarpal, opponens pollicis or palmar carpal ligament [14]. In some of the cases, the first lumbrical was found to have bipennate arrangement. In the present case, we report a rare case of additional slip of first lumbrical muscle from one of the aberrant tendon of the FPL. Unusual relationship between the additional tendon of FPL, additional slip of origin of first lumbrical and the FDS tendon for index finger observed in the present case reveal an important clue to the phylogenetic common origin of FDS [15].

The abnormal position of the tendon can affect the normal anatomy of the carpal tunnel and may lead to the compression of median nerve and subsequently result in carpal tunnel syndrome. In 1968, Entin categorized the structures that cause carpal tunnel syndrome into following groups; the structures that reduce the capacity of the tunnel, the structures that increase the volume of its contents and the structures that form part of a systemic condition [16]. The aberrant tendons and unusual origin of first lumbrical observed in the present case can compress the median nerve and cause the carpal tunnel syndrome by increasing the volume of the carpal tunnel. Earlier, it has been demonstrated that the presence of aberrant tendons is the common cause of compressive neuropathy of median nerve in the carpal tunnel [17]. Kiztan has reported a case of carpal tunnel syndrome caused by the anatomical variation of FDS tendon in the carpal tunnel [18].

Apart from the above mentioned clinical importance of the present case, the aberrant third tendon of FPL may pull the tendon of FDS for index finger during contraction of accessory belly of FPL. Also a slip of first lumbrical muscle originating from the aberrant tendon may alter the pulling direction of FDS tendon indirectly, thus altering the movements in the index finger.

Knowledge of anomalous tendons of the FPL reported in the present case is essentially important to avoid clinical complications during surgical approach of carpal tunnel. As the additional tendons muscle bellies and tendons can cause chronic inflammation, nerve compressions and tenosynovitis, the knowledge of this variation is also important for the orthopedic surgeons.

\section{REFERENCES}

[1] Williams, P.L., Bannister, L.H., Berry, M.M., Collins, P., Dyson, M., Dussek, J.E. and Ferguson, M.W. (1995) Gray's anatomy: The anatomical basis of medicine and surgery. 38th Edition, Churchill Livingstone, New York.

[2] Wood, J. (1868) Variations in human myology. Proceedings of the Royal Society of London, 15, 518-846.

[3] Dykes, J. and Anson, B.J. (1944) The accessory tendon of the flexor pollicis longus muscle. The Anatomical Record, 90, 83-87. http://dx.doi.org/10.1002/ar.1090900111

[4] Malhotra, V.K., Sing, N.P., Tewari, S.P. (1982) The accessory head of the flexor pollicis longus muscle and its nerve supply. Anatomischer Anzeiger, 151, 503-505.

[5] Jones, M., Abrahams, P.H., Sanudo, J.R., Campillo, M. (1997) Incidence and morphology of accessory heads of flexor pollicis longus and flexor digitorum profundus (Gantzer's muscle). Journal of Anatomy, 191, 451-455. http://dx.doi.org/10.1046/j.1469-7580.1997.19130451.x

[6] Hemmady, M.V., Subramanya, A.V. and Mehta, I.M. (1993) Occasional head of flexor pollicis longus muscle: A study of its morphology and clinical significance. Journal of Postgraduate Medicine, 39, 14-16.

[7] Mahakkanukrauh, P., Surin, P., Ongkana, N., Sethadavit, M. and Vaidhayakarn, P. (2004) Prevalence of accessory head of flexor pollicis longus muscle and its relation to anterior interosseus nerve in Thai population. Clinical Anatomy, 17, 631-635. http://dx.doi.org/10.1002/ca.20016

[8] Macalister, A. (1875) Additional observatins on muscular anomalies in human anatomy (third series), with a catalogue of the principal musclular variations hitherto published. Transactions of the Royal Irish Academy Science, 25, 1-134.

[9] Linburg, R.M. and Comstock, B.E. (1979) Anomalous tendon slips from the flexor pollicis longus to flexor digitorum profundus. The Journal of Hand Surgery, 4, 79-83. http://dx.doi.org/10.1016/S0363-5023(79)80110-0

[10] Vishal, K., Naveen, N.S., Murlimanju, B.V., Prima, S.D. (2011) A rare muscular variation in the flexor compartment of the forearm. The International Journal of Acoustics and Vibration, 4, 115-116.

[11] Jones, M., Abrahams, P.H. and Sanudo, J.R. (1997) Case report: Accessory head of the deep forearm flexors. Journal of Anatomy, 191, 313-314. http://dx.doi.org/10.1046/j.1469-7580.1997.19120313.x

[12] Lewis, W.H. (1910) The development of the muscular system. In: Keibel, F. and Mall, F.P., Eds., Manual of Human Embryology, J. B. Lippincott, Philadelphia, 492493. 
[13] Bergman, R.A., Thompson, S.A., Afifi, A.K. and Saadeh, F.A. (1988) Compendium of human anatomic variation. Urban and Schwarzenberg, Munich.

[14] Ajmani, M.L. (2001) Morphological variations of lumbrical muscles in the human hand with some observations on its nerve supply. Medical Journal of the Iranian Hospital, 3, 20-25.

[15] Koizumi, M., Kawai, K., Honma, S. and Kodma, K. (2002) Anomalous lumbrical muscles arising from the deep surface of flexor digitorum superficialis muscles in man. Annals of Anatomy, 184, 387-392.

http://dx.doi.org/10.1016/S0940-9602(02)80062-0
[16] Entin, M.A. (1968) Carpal tunnel syndrome and its variants. Surgical Clinics of North America, 48, 1097-1112.

[17] Ametewee, K., Harris, A. and Samuel, M. (1985) Acute carpal tunnel syndrome produced by anomalous flexor digitorum superficialis indicis muscle. Journal of Hand Surgery (European Volume), 10, 83-84. http://dx.doi.org/10.1016/S0266-7681(85)80025-5

[18] Kiztan, T. (1986) Carpal tunnel syndrome caused by a rare tendon variation. Handchirurgie, Mikrochirurgie, Plastische Chirurgie, 18, 77-78. 